Article

\title{
New Sharp Bounds for the Modified Bessel Function of the First Kind and Toader-Qi Mean ${ }^{\dagger}$
}

\author{
Zhen-Hang Yang ${ }^{1,2} \mathbb{D}$, Jing-Feng Tian ${ }^{3, *} \mathbb{D}$ and Ya-Ru Zhu ${ }^{3}$ \\ 1 Engineering Research Center of Intelligent Computing for Complex Energy Systems of Ministry of Education, \\ North China Electric Power University, Yonghua Street 619, Baoding 071003, China; yzhkm@163.com \\ 2 Zhejiang Society for Electric Power, Hangzhou 310014, China \\ 3 Department of Mathematics and Physics, North China Electric Power University, Yonghua Street 619, \\ Baoding 071003, China; zhuyaru1982@126.com \\ * Correspondence: tianjf@ncepu.edu.cn \\ + Dedicated to the 60th Anniversary of Zhejiang Electric Power Company Research Institute.
}

Received: 6 May 2020; Accepted: 29 May 2020; Published: 2 June 2020

Abstract: Let $I_{v}(x)$ be he modified Bessel function of the first kind of order $v$. We prove the double inequality $\sqrt{\frac{\sinh t}{t} \cosh ^{1 / q}(q t)}<I_{0}(t)<\sqrt{\frac{\sinh t}{t} \cosh ^{1 / p}(p t)}$ holds for $t>0$ if and only if $p \geq 2 / 3$ and $q \leq(\ln 2) / \ln \pi$. The corresponding inequalities for means improve already known results.

Keywords: modified Bessel function of the first kind; hyperbolic function; mean; inequality

MSC: 39B62; 33B10

\section{Introduction}

The modified Bessel function of the first kind of order $v$, denoted by $I_{v}(x)$, is a particular solution of the second-order differential equation ([1], p. 77)

$$
x^{2} y^{\prime \prime}(x)+x y^{\prime}(x)-\left(x^{2}+v^{2}\right) y(x)=0,
$$

which can be represented explicitly by the infinite series as

$$
I_{v}(x)=\sum_{n=0}^{\infty} \frac{(x / 2)^{2 n+v}}{n ! \Gamma(v+n+1)}, x \in \mathbb{R}, v \in \mathbb{R} \backslash\{-1,-2, \ldots\},
$$

where $\Gamma(x)$ is the gamma function [2-4]. There are many properties of $I_{v}(x)$, see for example, [5-11].

In this paper, we are interested in a special case of $I_{v}(x)$, that is, $I_{0}(x)$, which is related to Toader-Qi mean of positive numbers $a$ and $b$ defined by

$$
T Q(a, b)=\frac{2}{\pi} \int_{0}^{\pi / 2} a^{\cos ^{2} \theta} b^{\sin ^{2} \theta} d \theta=\sqrt{a b} I_{0}\left(\ln \sqrt{\frac{a}{b}}\right)
$$

(see [12-14]), where and in what follows $a, b>0$ with $a \neq b$. It is undoubted that Toader-Qi mean $T Q(a, b)$ is a new newcomer. Recall that some classical means including the arithmetic mean, geometric mean, logarithmic mean, exponential mean and power mean of order $p$ defined by

$$
\begin{array}{ll}
A \equiv A(a, b)=\frac{a+b}{2}, & G \equiv G(a, b)=\sqrt{a b}, \\
L \equiv L(a, b)=\frac{a-b}{\ln a-\ln b}, & I \equiv I(a, b)=e^{-1}\left(\frac{b^{b}}{a^{a}}\right)^{1 /(b-a)},
\end{array}
$$




$$
A_{p} \equiv A_{p}(a, b)=\left(\frac{a^{p}+b^{p}}{2}\right)^{1 / p} \text { if } p \neq 0 \text { and } A_{0} \equiv A_{0}(a, b)=\sqrt{a b}
$$

respectively. Clearly, $A(a, b)=A_{1}(a, b)$ and $G(a, b)=A_{0}(a, b)$. It is known that $p \mapsto A_{p}(a, b)$ is increasing on $\mathbb{R}$. A simple relation among these elementary means is the following inequalities:

$$
G<L<A_{1 / 3}<\frac{A+2 G}{3}<A_{1 / 2}<\frac{2 A+G}{3}<A_{2 / 3}<I<A_{\ln 2}<A_{1}
$$

(see [15-21]). Another interesting relation proven in [22] is that:

$$
\sqrt{A G}<\sqrt{L I}<\frac{L+I}{2}<\frac{A+G}{2} .
$$

Let $b>a>0$ and $t=\ln \sqrt{a / b}$. Then those means mentioned above can be represented in terms of hyperbolic functions:

$$
\begin{aligned}
\frac{L(a, b)}{\sqrt{a b}} & =\frac{\sinh t}{t}, \frac{I(a, b)}{\sqrt{a b}}=\exp \left(\frac{t}{\tanh t}-1\right), \\
\frac{T Q(a, b)}{\sqrt{a b}} & =I_{0}(t), \quad \frac{A_{p}(a, b)}{\sqrt{a b}}=\cosh ^{1 / p}(p t) \text { for } p \neq 0 .
\end{aligned}
$$

Correspondingly, the inequalities mentioned above are equivalent to

$$
\begin{aligned}
1 & <\frac{\sinh t}{t}<\cosh ^{3}\left(\frac{t}{3}\right)<\frac{\cosh t+2}{3}<\cosh ^{2}\left(\frac{t}{2}\right) \\
& <\frac{2 \cosh t+1}{3}<\cosh ^{3 / 2}\left(\frac{2 t}{3}\right)<\exp \left(\frac{t}{\tanh t}-1\right)<\cosh ^{1 / \ln 2}(t \ln 2) \\
\sqrt{\cosh t} & <\sqrt{\frac{\sinh t}{t} \exp \left(\frac{t}{\tanh t}-1\right)}<\frac{1}{2}\left[\frac{\sinh t}{t}+\exp \left(\frac{t}{\tanh t}-1\right)\right]<\frac{\cosh t+1}{2} .
\end{aligned}
$$

for $t>0$.

Let us return to Toader-Qi mean. In 2015, Qi, Shi, Liu and Yang [13] proved that the inequalities

$$
L(a, b)<T Q(a, b)<\frac{A(a, b)+G(a, b)}{2}<\frac{2 A(a, b)+G(a, b)}{3}<I(a, b)
$$

hold. Yang and Chu (Theorem 3.3 of [23]) established a series of sharp inequalities for $T Q(a, b)$ and $I_{0}(t)$, for example, the inequalities

$$
\begin{gathered}
\sqrt{\frac{\sinh (2 t)}{\pi t}}<I_{0}(t)<\sqrt{\frac{\sinh (2 t)}{2 t}}, \\
\sqrt{\left(\frac{2}{\pi} \cosh t+1-\frac{2}{\pi}\right) \frac{\sinh t}{t}}<I_{0}(t)<\sqrt{\left(\lambda_{0} \cosh t+1-\lambda_{0}\right) \frac{\sinh t}{t}} \\
\left(\frac{\sinh t}{t}\right)^{3 / 4}(\cosh t)^{1 / 4}<I_{0}(t)<\frac{3 \sinh t}{4}+\frac{1}{4} \cosh t
\end{gathered}
$$

hold for $t>0$ with $\lambda_{0}=0.6766 \ldots$ Inspired by the inequalities (3) and (4), Yang and Chu conjectured further that the inequality

$$
T Q(a, b)<\sqrt{L(a, b) I(a, b)}
$$


holds, which was proven in Theorem 3.1 of [24] by Yang, Chu and Song. In fact, they proved the following double inequality

$$
\sqrt{\frac{e}{\pi}} \sqrt{L(a, b) I(a, b)}<T Q(a, b)<\sqrt{L(a, b) I(a, b)}
$$

holds with the best coefficients $\sqrt{e / \pi}=0.930 \ldots$ and 1 . More inequalities for $T Q(a, b)$ can be seen in $[25,26]$.

Motivated by the inequalities (9) and $A_{2 / 3}<I$ listed in (3), the aim of this paper is to find the best constants $p$ and $q$ such that double inequality

$$
\sqrt{L(a, b) A_{q}(a, b)}<T Q(a, b)<\sqrt{L(a, b) A_{p}(a, b)}
$$

holds, or equivalently,

$$
\sqrt{\frac{\sinh t}{t} \cosh ^{1 / q}(q t)}<I_{0}(t)<\sqrt{\frac{\sinh t}{t} \cosh ^{1 / p}(p t)}
$$

for $t>0$. Our main results are as follows.

Theorem 1. The function

$$
F(t)=\frac{t I_{0}(t)^{2}}{\cosh ^{3 / 2}(2 t / 3) \sinh t}
$$

is strictly decreasing from $(0, \infty)$ onto $(\sqrt{8} / \pi, 1)$. Therefore, the double inequality

$$
\frac{2^{3 / 4}}{\sqrt{\pi}} \sqrt{\frac{\sinh t}{t} \cosh ^{3 / 2}\left(\frac{2 t}{3}\right)}<I_{0}(t)<\sqrt{\frac{\sinh t}{t} \cosh ^{3 / 2}\left(\frac{2 t}{3}\right)}
$$

holds for $t>0$, or equivalently,

$$
\frac{2^{3 / 4}}{\sqrt{\pi}} \sqrt{L(a, b) A_{2 / 3}(a, b)}<T Q(a, b)<\sqrt{L(a, b) A_{2 / 3}(a, b)}
$$

holds, where the coefficients $2^{3 / 4} / \sqrt{\pi}=0.94885 \ldots$ and 1 are the best.

Theorem 2. The double inequality (12) holds for $t>0$, or equivalently, (11) holds for $a, b>0$ with $a \neq b$, if and only if $p \geq 2 / 3$ and $q \leq p_{0}=(\ln 2) / \ln \pi=0.605 \ldots$.

\section{Tools and Lemmas}

To prove our results, we need two tools. The first tool was due to Biernacki and Krzyz [27], which play an important role in dealing with the monotonicity of the ratio of power series.

Lemma 1 ( [27]). Let $A(t)=\sum_{k=0}^{\infty} a_{k} t^{k}$ and $B(t)=\sum_{k=0}^{\infty} b_{k} t^{k}$ be two real power series converging on $(-r, r)$ $(r>0)$ with $b_{k}>0$ for all $k$. If the sequence $\left\{a_{k} / b_{k}\right\}$ is increasing (decreasing) for all $k$, then the function $t \mapsto A(t) / B(t)$ is also increasing (decreasing) on $(0, r)$.

Remark 1. Recently, another monotonicity rule in the case when the sequence $\left\{a_{k} / b_{k}\right\}_{k \geq 0}$ is piecewise monotonic was presented in Theorem 1 of [28], which is now applied preliminarily, see for example, [29-32].

The second tool is the so-called "L'Hospital Monotone Rule" (or, for short, LMR), which is very effective in studying the monotonicity of ratios of two functions. 
Lemma 2 ([33], Theorem 2). Let $-\infty<a<b<\infty$, and let $f, g:[a, b] \rightarrow \mathbb{R}$ be continuous functions that are differentiable on $(a, b)$, with $f(a)=g(a)=0$ or $f(b)=g(b)=0$. Assume that $g^{\prime}(x) \neq 0$ for each $x$ in $(a, b)$. If $f^{\prime} / g^{\prime}$ is increasing (decreasing) on $(a, b)$ then so is $f / g$.

The following two lemmas will be used to prove Proposition 1.

Lemma 3 ([23], Lemma 2.8). We have

$$
I_{0}(t)^{2}=\sum_{n=0}^{\infty} \frac{(2 n) !}{2^{2 n} n ! !^{4}} t^{2 n}
$$

Lemma 4 ([34], Problems 85, 94). The two given sequences $\left\{a_{n}\right\}_{n \geq 0}$ and $\left\{b_{n}\right\}_{n \geq 0}$ satisfy the conditions

$$
b_{n}>0 ; \sum_{n=0}^{\infty} b_{n} t^{n} \text { converges for all values of } t ; \lim _{n \rightarrow \infty} \frac{a_{n}}{b_{n}}=s \text {. }
$$

Then $\sum_{n=0}^{\infty} a_{n} t^{n}$ converges too for all values of $t$ and in addition

$$
\lim _{t \rightarrow \infty} \frac{\sum_{n=0}^{\infty} a_{n} t^{n}}{\sum_{n=0}^{\infty} b_{n} t^{n}}=s
$$

\section{Three Propositions}

The proofs of Theorems 1 and 2 rely on the following propositions.

Proposition 1. Let

$$
f_{0}(t)=\theta \frac{2 \cosh t+1}{3} \frac{\sinh t}{t}+(1-\theta)\left(1+\frac{1}{2} t^{2}+\frac{229}{6720} t^{4}\right),
$$

where $\theta=11,009 / 10,449$. The function

$$
F_{0}(t)=\frac{I_{0}(t)^{2}}{f_{0}(t)}
$$

is strictly decreasing from $(0, \infty)$ onto $(3 /(\theta \pi), 1)$.

Proof. Expanding in power series yields

$$
\begin{aligned}
f_{0}(t) & =\theta \frac{\sinh 2 t+\sinh t}{3 t}+(1-\theta)\left(1+\frac{1}{2} t^{2}+\frac{229}{6720} t^{4}\right) \\
& =\theta \sum_{n=0}^{\infty} \frac{2^{2 n+1}+1}{3(2 n+1) !} t^{2 n}+(1-\theta)\left(1+\frac{1}{2} t^{2}+\frac{229}{6720} t^{4}\right) \\
& =1+\frac{1}{2} t^{2}+\frac{387 \theta+229}{6720} t^{4}+\sum_{n=3}^{\infty} \frac{\theta\left(2^{2 n+1}+1\right)}{3(2 n+1) !} t^{2 n}:=\sum_{n=0}^{\infty} v_{n} t^{2 n},
\end{aligned}
$$

where $v_{0}=1, v_{1}=1 / 2$,

$$
v_{2}=\frac{387 \theta+229}{6720} \text { and } v_{n}=\frac{\theta\left(2^{2 n+1}+1\right)}{3(2 n+1) !} \text { for } n \geq 3 .
$$

By Lemma 3, we see that

$$
I_{0}(t)^{2}=\sum_{n=0}^{\infty} \frac{(2 n) !}{2^{2 n} n !^{4}} t^{2 n}:=\sum_{n=0}^{\infty} u_{n} t^{2 n}
$$


Direct calculations gives

$$
\begin{aligned}
& \frac{u_{0}}{v_{0}}=\frac{u_{1}}{v_{1}}=1, \frac{u_{2}}{v_{2}}=\frac{630}{387 \theta+229} \\
& \frac{u_{n}}{v_{n}}=\frac{(2 n) !}{2^{2 n} n !^{4}} / \frac{\theta\left(2^{2 n+1}+1\right)}{3(2 n+1) !} \text { for } n \geq 3,
\end{aligned}
$$

then

$$
\begin{gathered}
\frac{u_{1}}{v_{1}}-\frac{u_{0}}{v_{0}}=0, \quad \frac{u_{2}}{v_{2}}-\frac{u_{1}}{v_{1}}=-\frac{387 \theta-401}{387 \theta+229}<0, \\
\frac{u_{3}}{v_{3}}-\frac{u_{2}}{v_{2}}=-\frac{35}{172} \frac{1161 \theta-1145}{\theta(387 \theta+229)}<0, \\
\frac{u_{n+1}}{v_{n+1}} / \frac{u_{n}}{v_{n}}-1=-\frac{2^{2 n+1}-\left(3 n^{2}+6 n+2\right)}{(n+1)^{2}\left(2^{2 n+3}+1\right)}<0 \text { for } n \geq 3,
\end{gathered}
$$

where the last inequality holds due to

$$
\begin{aligned}
2^{2 n+1}-\left(3 n^{2}+6 n+2\right) & >1+(2 n+1)+\frac{(2 n+1)(2 n)}{2 !}+\frac{(2 n+1)(2 n)(2 n-1)}{3 !} \\
-\left(3 n^{2}+6 n+2\right) & =\frac{1}{3} n(4 n+5)(n-2)>0 \text { for } n \geq 3 .
\end{aligned}
$$

This shows that the sequence $\left\{u_{n} / v_{n}\right\}_{n \geq 0}$ is strictly decreasing, so is $I_{0}(t)^{2} / f_{0}(t)$ on $(0, \infty)$ by Lemma 1 . It is easy to check that

$$
\lim _{t \rightarrow 0} \frac{I_{0}(t)^{2}}{f_{0}(t)}=\frac{u_{0}}{v_{0}}=1 \text { and } \lim _{t \rightarrow \infty} \frac{I_{0}(t)^{2}}{f_{0}(t)}=\lim _{n \rightarrow \infty} \frac{u_{n}}{v_{n}}=\frac{3}{\pi \theta},
$$

where the second limits holds due to Lemma 4, thereby completing the proof.

Proposition 2. Let $f_{0}(t)$ be defined by (15). The function

$$
F_{1}(t)=\frac{t f_{0}(t)}{\cosh ^{3 / 2}(2 t / 3) \sinh t}
$$

is strictly decreasing from $(0, \infty)$ onto $(\sqrt{8} \theta / 3,1)$, where $\theta=11,009 / 10,449$.

Proof. Let

$$
\begin{aligned}
f_{1}(t)= & \ln F_{1}(t)=\ln \left[\theta \frac{2 \cosh t+1}{3} \frac{\sinh t}{t}+(1-\theta)\left(1+\frac{1}{2} t^{2}+\frac{229}{6720} t^{4}\right)\right] \\
& -\frac{3}{2} \ln \left(\cosh \frac{2 t}{3}\right)-\ln \frac{\sinh t}{t} .
\end{aligned}
$$

Differentiation yields

$$
f_{1}^{\prime}(t)=-\frac{1}{6 t \sinh t \cosh (2 t / 3)} \frac{f_{2}(t)}{f_{0}(t)}
$$

where

$$
f_{2}(t)=t^{5} f_{25}(t)+t^{4} f_{24}(t)+t^{3} f_{23}(t)+t^{2} f_{22}(t)+t f_{21}(t)+f_{20}(t),
$$




$$
\begin{aligned}
f_{25}(t)= & \frac{229}{1120}(1-\theta)\left(\cosh \frac{2 t}{3} \cosh t+3 \sinh \frac{2 t}{3} \sinh t\right) \\
f_{24}(t)= & \frac{229}{224}(\theta-1) \cosh \frac{2 t}{3} \sinh t \\
f_{23}(t)= & \frac{3}{2}(1-\theta)\left(2 \cosh \frac{2 t}{3} \cosh t+3 \sinh \frac{2 t}{3} \sinh t\right) \\
f_{22}(t)= & 9(\theta-1) \cosh \frac{2 t}{3} \sinh t \\
f_{21}(t)= & 3(1-\theta)\left(2 \cosh \frac{2 t}{3} \cosh t+3 \sinh \frac{2 t}{3} \sinh t\right) \\
f_{20}(t)= & 6(\theta-1) \cosh \frac{2 t}{3} \sinh t-4 \theta \cosh \frac{2 t}{3} \sinh ^{3} t \\
& +3 \theta \sinh \frac{2 t}{3} \sinh ^{2} t+6 \theta \sinh \frac{2 t}{3} \cosh t \sinh ^{2} t .
\end{aligned}
$$

Expanding in power series gives

$$
\begin{aligned}
& f_{25}(3 s)=-\frac{229}{4480}(\theta-1)(5 \cosh 5 s-\cosh s)=-\frac{229}{4480}(\theta-1) \sum_{n=2}^{\infty} \frac{5^{2 n-3}-1}{(2 n-4) !} s^{2 n-4}, \\
& f_{24}(3 s)=\frac{229}{448}(\theta-1)(\sinh 5 s+\sinh s)=\frac{229}{448}(\theta-1) \sum_{n=2}^{\infty} \frac{5^{2 n-3}+1}{(2 n-3) !} s^{2 n-3}, \\
& f_{23}(3 s)=-\frac{3}{4}(\theta-1)(5 \cosh 5 s-\cosh s)=-\frac{3}{4}(\theta-1) \sum_{n=1}^{\infty} \frac{5^{2 n-1}-1}{(2 n-2) !} s^{2 n-2}, \\
& f_{22}(3 s)=\frac{9}{2}(\theta-1)(\sinh 5 s+\sinh s)=\frac{9}{2}(\theta-1) \sum_{n=1}^{\infty} \frac{5^{2 n-1}+1}{(2 n-1) !} s^{2 n-1}, \\
& f_{21}(3 s)=-\frac{3}{2}(\theta-1)(5 \cosh 5 s-\cosh s)=-\frac{3}{2}(\theta-1) \sum_{n=0}^{\infty} \frac{5^{2 n+1}-1}{(2 n) !} s^{2 n}, \\
& f_{20}(3 s)=\frac{1}{4} \theta \sinh 11 s+\frac{3}{4} \theta \sinh 8 s-\frac{5}{4} \theta \sinh 7 s+\left(\frac{15}{4} \theta-3\right) \sinh 5 s \\
& -\frac{3}{4} \theta \sinh 4 s-\frac{3}{2} \theta \sinh 2 s+\left(\frac{21}{4} \theta-3\right) \sinh s \\
& =\sum_{n=0}^{\infty}\left[\frac{\theta}{4} 11^{2 n+1}+\frac{3 \theta}{4} 8^{2 n+1}-\frac{5 \theta}{4} 7^{2 n+1}+\left(\frac{15 \theta}{4}-3\right) 5^{2 n+1}\right. \\
& \left.-\frac{3 \theta}{4} 4^{2 n+1}-\frac{3 \theta}{2} 2^{2 n+1}+\left(\frac{21}{4} \theta-3\right)\right] \frac{s^{2 n+1}}{(2 n+1) !}
\end{aligned}
$$

Then $f_{2}(3 s)$ defined by (16) can be written as

$$
\begin{aligned}
f_{2}(3 s) & =243 s^{5} f_{25}(3 s)+81 s^{4} f_{24}(3 s)+27 s^{3} f_{23}(3 s)+9 s^{2} f_{22}(3 s)+3 s f_{21}(3 s)+f_{20}(3 s) \\
& =54 s^{3}+(\theta-1) \sum_{n=2}^{\infty} a_{n}^{[1]} \frac{s^{2 n+1}}{(2 n+1) !}+\sum_{n=2}^{\infty} a_{n}^{[2]} s^{2 n+1}+\frac{3 \theta}{4} \sum_{n=2}^{\infty} a_{n}^{[3]} \frac{s^{2 n+1}}{(2 n+1) !},
\end{aligned}
$$


where

$$
\begin{aligned}
a_{n}^{[1]}= & -\frac{55,647}{4480} \frac{(2 n+1) !}{(2 n-4) !} 5^{2 n-3}+\frac{18,549}{448} \frac{(2 n+1) !}{(2 n-3) !} 5^{2 n-3} \\
& -\frac{81}{4} \frac{(2 n+1) !}{(2 n-2) !} 5^{2 n-1}+\frac{81}{2} \frac{(2 n+1) !}{(2 n-1) !} 5^{2 n-1} \\
& -\frac{9}{2} \frac{(2 n+1) !}{(2 n) !} 5^{2 n+1}+\frac{3}{4} \frac{5 \theta-4}{\theta-1} 5^{2 n+1}+\frac{1}{4} \frac{\theta}{\theta-1} 11^{2 n+1}, \\
a_{n}^{[2]}= & \frac{55,647}{4480} \frac{\theta-1}{(2 n-4) !}+\frac{18,549}{448} \frac{\theta-1}{(2 n-3) !}+\frac{81}{4} \frac{\theta-1}{(2 n-2) !} \\
& +\frac{81}{2} \frac{\theta-1}{(2 n-1) !}+\frac{9}{2} \frac{\theta-1}{(2 n) !}+\frac{13(7 \theta-4)}{4} \frac{(2 n+1) !}{(2 n)} \\
a_{n}^{[3]}= & 8^{2 n+1}-\frac{5}{3} \times 7^{2 n+1}-4^{2 n+1}-2^{2 n+2} .
\end{aligned}
$$

It remains to prove $a_{n}^{[i]}>0$ for $i=1,2,3$ and $n \geq 2$. It is clear that $a_{n}^{[2]}>0$ due to $\theta=$ $11,009 / 10,449>1$. For $a_{n}^{[3]}$, it is easy to check that

$$
a_{n+1}^{[3]}-49 a_{n}^{[3]}=12\left(10 \times 2^{4 n}+11 \times 2^{2 n}+15\right) \times 2^{2 n}>0,
$$

which together with $a_{2}^{[3]}=11,005>0$ yields $a_{n}^{[3]}>0$ for all $n \geq 2$. For $a_{n}^{[1]}$, since $(5 \theta-4)>$ $5(\theta-1)$ and

$$
\frac{\theta}{\theta-1}=\frac{11,009}{560}=19.659 \ldots>18
$$

we have

$$
\begin{aligned}
a_{n}^{[1]}> & -\frac{55,647}{4480} \frac{(2 n+1) !}{(2 n-4) !} 5^{2 n-3}+\frac{18,549}{448} \frac{(2 n+1) !}{(2 n-3) !} 5^{2 n-3} \\
& -\frac{81}{4} \frac{(2 n+1) !}{(2 n-2) !} 5^{2 n-1}+\frac{81}{2} \frac{(2 n+1) !}{(2 n-1) !} 5^{2 n-1} \\
& -\frac{9}{2} \frac{(2 n+1) !}{(2 n) !} 5^{2 n+1}+\frac{15}{4} \times 5^{2 n+1}+\frac{9}{2} \times 11^{2 n+1} \\
= & \frac{9}{2} \times 11^{2 n+1}-3\left(\frac{18,549}{28} n^{5}-\frac{154,575}{56} n^{4}+\frac{138,915}{16} n^{3}\right. \\
& \left.-\frac{1,357,425}{224} n^{2}+\frac{848,523}{224} n+\frac{3125}{4}\right) \times 5^{2 n-4}:=a_{n}^{[0]} .
\end{aligned}
$$

The sequence $\left\{a_{n}^{[0]}\right\}_{n \geq 2}$ satisfies the recurrence relation

$$
\begin{aligned}
\frac{a_{n+1}^{[0]}-121 a_{n}^{[0]}}{9 \times 5^{2 n-4}}= & \frac{148,392}{7} n^{5}-\frac{463,725}{4} n^{4}+\frac{2,202,435}{7} n^{3} \\
& -\frac{36,754,425}{112} n^{2}+\frac{3,895,809}{56} n-\frac{21,875}{2},
\end{aligned}
$$


which can be written as

$$
\begin{aligned}
& \frac{148,392}{7}(n-2)^{5}+\frac{2,689,605}{28}(n-2)^{4}+\frac{1,645,965}{7}(n-2)^{3} \\
& +\frac{52,997,895}{112}(n-2)^{2}+\frac{29,042,799}{56}(n-2)+\frac{312,145}{2}>0
\end{aligned}
$$

for $n \geq 2$. This in combination with $a_{2}^{[0]}=10,126,407 / 16>0$ leads to $a_{n}^{[0]}>0$ for $n \geq 2$, and so is $a_{n}^{[1]}$. Therefore, $f_{1}(t)>0$ for $t>0$, so $f_{0}(t)$ is strictly increasing on $(0, \infty)$. An easy computation yields

$$
\lim _{t \rightarrow 0} f_{1}(t)=0 \text { and } \lim _{t \rightarrow \infty} f_{1}(t)=\ln \frac{\sqrt{8} \theta}{3}
$$

which completes the proof.

Using Lemma 2 we can prove the following lemma, which will be use to prove Theorem 2.

Proposition 3. Let $q \neq 0,1 / 2,1$. The ratio

$$
t \mapsto \frac{\cosh ^{1 / q}(q t)-1}{\cosh t-1}
$$

is strictly increasing on $(0, \infty)$ if $q \in(-\infty, 0) \cup(1 / 2,1)$ and strictly decreasing on $(0, \infty)$ if $q \in(0,1 / 2) \cup$ $(1, \infty)$. Consequently, the double inequality

$$
q \cosh t+1-q<\cosh ^{1 / q}(q t)<c_{q} \cosh t+1-c_{q}
$$

holds for $t>0$ if $q \in(-\infty, 0) \cup(1 / 2,1)$, where the weights $q$ and $c_{q}=2^{1-1 / q}$ if $q>0$ and $c_{q}=0$ if $q<0$ are the best possible. If $q \in(0,1 / 2) \cup(1, \infty)$, then the double inequality (17) is reversed.

Proof. Let

$$
g_{1}(t)=\cosh ^{1 / q}(q t)-1 \text { and } g_{2}(t)=\cosh t-1 .
$$

Clearly, $g_{1}\left(0^{+}\right)=g_{2}\left(0^{+}\right)=0$, and

$$
\lim _{t \rightarrow 0} \frac{g_{1}(t)}{g_{2}(t)}=q \text { and } \lim _{t \rightarrow \infty} \frac{g_{1}(t)}{g_{2}(t)}=c_{q}=\left\{\begin{array}{cl}
2^{1-1 / q} & \text { if } q>0 \\
0 & \text { if } q<0 .
\end{array}\right.
$$

Differentiation yields

$$
\begin{aligned}
\frac{g_{1}^{\prime}(t)}{g_{2}^{\prime}(t)} & =\frac{\cosh ^{1 / q-1}(q t) \sinh (q t)}{\sinh t} \\
{\left[\frac{g_{1}^{\prime}(t)}{g_{2}^{\prime}(t)}\right]^{\prime} } & =\frac{(1-2 q) t}{2 \sinh ^{2} t \cosh ^{2-1 / q}(q t)}\left(\frac{\sinh |(1-2 q) t|}{|(1-2 q) t|}-\frac{\sinh t}{t}\right) .
\end{aligned}
$$

Since the function $(\sinh x) / x$ is strictly increasing on $(0, \infty)$, we find that

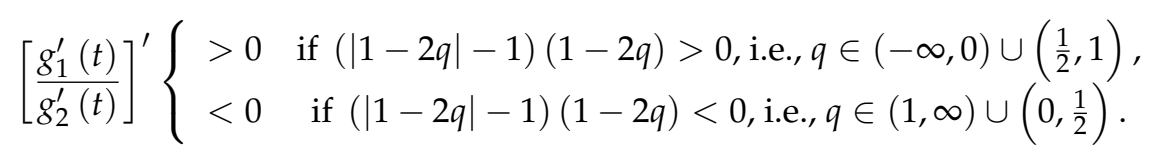

By Lemma 2, the desired monotonicity follows. The double inequality (17) and its reverse follow from the monotonicity of $g_{1}(t) / g_{2}(t)$ on $(0, \infty)$. This completes the proof. 
Remark 2. Taking $q=p_{0}=(\ln 2) / \ln \pi$ in the double inequality (17) we obtain the double inequality

$$
p_{0} \cosh t+1-p_{0}<\cosh ^{1 / p_{0}}\left(p_{0} t\right)<\frac{2}{\pi} \cosh t+1-\frac{2}{\pi}
$$

for $t>0$.

Remark 3. The generalized Heronian mean [35] is defined by

$$
H_{w}(a, b)=\frac{a+b+w \sqrt{a b}}{w+2} .
$$

Let $t=\ln \sqrt{a / b}$ with $b>a>0$ and $q=w /(w+2)>0$. Then Proposition 3 give a best approximation for $H_{w}(a, b)$ by power means:

$$
\begin{aligned}
& H_{w}(a, b)<A_{w /(w+2)}(a, b) \text { if } w \in(2, \infty) \\
& H_{w}(a, b)>A_{w /(w+2)}(a, b) \text { if } q \in(0,2) .
\end{aligned}
$$

Our proof is clearly concise than Li, Long and Chu's given in [35].

\section{Proofs of Theorem 1 and 2}

We are now in a position to prove Theorems 1 and 2.

Proof of Theorem 1. We have

$$
F(t)=\frac{t I_{0}(t)^{2}}{\cosh ^{3 / 2}(2 t / 3) \sinh t}=\frac{I_{0}(t)^{2}}{f_{0}(t)} \times \frac{t f_{0}(t)}{\cosh ^{3 / 2}(2 t / 3) \sinh t}=F_{0}(t) \times F_{1}(t) .
$$

As shown in Propositions 1 and 2, the functions $F_{0}(t)$ and $F_{1}(t)$ are both strictly positive and decreasing on $(0, \infty)$, so is $F(t)$. And, we easily obtain

$$
\begin{aligned}
& \lim _{t \rightarrow 0} F(t)=\lim _{t \rightarrow 0} F_{0}(t) \times \lim _{t \rightarrow 0} F_{1}(t)=1, \\
& \lim _{t \rightarrow \infty} F(t)=\lim _{t \rightarrow \infty} F_{0}(t) \times \lim _{t \rightarrow \infty} F_{1}(t)=\frac{3}{\pi \theta} \frac{\sqrt{8} \theta}{3}=\frac{\sqrt{8}}{\pi} .
\end{aligned}
$$

Using the monotonicity of $F(t)$, the desired double inequality follows. This completes the proof.

Proof of Theorem 2. (i) The necessary condition for the right hand side inequality of (12) to hold follows from the limit relation

$$
\lim _{t \rightarrow 0} \frac{I_{0}(t)^{2}-\cosh ^{1 / p}(p t)(\sinh t) / t}{t^{2}}=-\frac{1}{6}(3 p-2) \leq 0 .
$$

The sufficiency follow from Theorem 1 and the increasing property of $p \mapsto \cosh ^{1 / p}(p t)$ on $\mathbb{R}$.

(ii) The necessary condition for the left hand side inequality of (12) to hold follows from the limit relation

$$
\lim _{t \rightarrow \infty} \frac{(\cosh (q t))^{1 / q}(\sinh t) / t}{I_{0}(t)^{2}} \leq 1
$$

Since $I_{0}(t) \sim e^{t} / \sqrt{2 \pi t}$ as $t \rightarrow \infty($ see $[36], 9.7 .1)$ and

$$
\begin{aligned}
& \cosh ^{1 / q}(q t) \frac{\sinh t}{t} \leq \frac{\sinh t}{t} \sim \frac{e^{t}}{2 t} \text { if } q \leq 0 \\
& \cosh ^{1 / q}(q t) \frac{\sinh t}{t}=e^{t}\left(\frac{1+e^{-2 q t}}{2}\right)^{1 / q} e^{t} \frac{1-e^{-2 t}}{2 t} \sim \frac{1}{2^{1 / q}} \frac{e^{2 t}}{2 t}
\end{aligned}
$$


we have

$$
\lim _{t \rightarrow \infty} \frac{\cosh ^{1 / q}(q t)(\sinh t) / t}{I_{0}(t)^{2}}=\left\{\begin{array}{cl}
0 & \text { if } q \leq 0, \\
\frac{\pi}{2^{1 / q}} & \text { if } q>0 .
\end{array}\right.
$$

Therefore, the necessary condition is that $\pi / 2^{1 / q} \leq 1$ if $q>0$ and $q \leq 0$, that is, $q \leq(\ln 2) / \ln \pi=p_{0}$.

By the increasing property of $q \mapsto \cosh ^{1 / q}(q t)$, to prove the sufficiency, it suffices to prove the left hand side inequality of (12) holds when $q=p_{0}$. From the first inequality of (7) and the second inequality of (18) it follows that

$$
I_{0}(t)>\sqrt{\frac{\sinh t}{t}\left(\frac{2}{\pi} \cosh t+1-\frac{2}{\pi}\right)}>\sqrt{\frac{\sinh t}{t} \cosh ^{1 / p_{0}}\left(p_{0} t\right)}
$$

for $t>0$, which proves the sufficiency, and the proof is completed.

\section{Concluding Remarks}

In this paper, we obtained the best constants $p$ and $q$ such that the double inequality (12) holds for $t>0$, or equivalently, (11) holds for $a, b>0$ with $a \neq b$. This improved the result in [24]. We close the paper by giving two remarks on our results.

Remark 4. It was shown in ([20], 5.25) that

$$
A_{2 / 3}(a, b)<I(a, b)<\frac{2 \sqrt{2}}{e} A_{2 / 3}(a, b) .
$$

Then the double inequality (11) can be extended as

$$
\begin{aligned}
\sqrt{\frac{e}{\pi}} \sqrt{L(a, b) I(a, b)} & <\frac{2^{3 / 4}}{\sqrt{\pi}} \sqrt{L(a, b) A_{2 / 3}(a, b)}<T Q(a, b) \\
& <\sqrt{L(a, b) A_{2 / 3}(a, b)}<\sqrt{L(a, b) I(a, b)} .
\end{aligned}
$$

Remark 5. As a computable bound, the upper bound $\sqrt{t^{-1} \sinh t \cosh ^{3 / 2}(2 t / 3)}$ for $I_{0}(t)$ is superior to those given (6) and (8). In fact, we have

$$
I_{0}(t)<\sqrt{\frac{\sinh t}{t} \cosh ^{3 / 2}\left(\frac{2 t}{3}\right)}<\sqrt{\frac{\sinh t}{t} \cosh t}=\sqrt{\frac{\sinh (2 t)}{2 t}}
$$

and

$$
I_{0}(t)<\sqrt{\frac{\sinh t}{t} \cosh ^{3 / 2}\left(\frac{2 t}{3}\right)}<\frac{3}{4} \frac{\sinh t}{t}+\frac{1}{4} \cosh t
$$

for $t>0$. The inequalities (19) are clear, and we have to check (20). Let

$$
h(t)=\ln \sqrt{\frac{\sinh (3 t / 2)}{3 t / 2} \cosh ^{3 / 2}(t)}-\ln \left[\frac{3}{4} \frac{\sinh (3 t / 2)}{3 t / 2}+\frac{1}{4} \cosh (3 t / 2)\right] .
$$

Differentiation yields

$$
h^{\prime}(t)=\frac{1}{6} \frac{h_{1}(t)}{t[\sinh (3 t)+t \cosh (3 t)] \cosh (2 t) \sinh (3 t)},
$$


where

$$
\begin{aligned}
h_{1}(t)= & t^{2}\left(3 \cosh 2 t \cosh ^{2} 3 t+3 \sinh 2 t \cosh 3 t \sinh 3 t-6 \cosh 2 t \sinh ^{2} 3 t\right) \\
& +\left(3 \sinh 2 t \sinh ^{2} 3 t-4 \cosh 2 t \cosh 3 t \sinh 3 t\right) t+\cosh 2 t \sinh ^{2} 3 t .
\end{aligned}
$$

Using "product into sum" formulas for hyperbolic functions and expanding in power series give

$$
\begin{aligned}
h_{1}(t)= & \frac{9}{2} t^{2} \cosh 2 t-\frac{3}{2} t^{2} \cosh 4 t-\frac{3}{2} t \sinh 2 t-\frac{7}{4} t \sinh 4 t-\frac{1}{4} t \sinh 8 t \\
& +\frac{1}{4} \cosh 4 t-\frac{1}{2} \cosh 2 t+\frac{1}{4} \cosh 8 t \\
h_{1}(t)= & \frac{9}{2} \sum_{n=1}^{\infty} \frac{2^{2 n-2}}{(2 n-2) !} t^{2 n}-\frac{3}{2} \sum_{n=1}^{\infty} \frac{4^{2 n-2}}{(2 n-2) !} t^{2 n} \\
& -\frac{3}{2} \sum_{n=1}^{\infty} \frac{2^{2 n-1}}{(2 n-1) !} t^{2 n}-\frac{7}{4} \sum_{n=1}^{\infty} \frac{4^{2 n-1}}{(2 n-1) !} t^{2 n} \\
& -\frac{1}{4} \sum_{n=1}^{\infty} \frac{8^{2 n-1}}{(2 n-1) !} t^{2 n}+\frac{1}{4} \sum_{n=0}^{\infty} \frac{4^{2 n}}{(2 n) !} t^{2 n} \\
& -\frac{1}{2} \sum_{n=0}^{\infty} \frac{2^{2 n}}{(2 n) !} t^{2 n}+\frac{1}{4} \sum_{n=0}^{\infty} \frac{8^{2 n}}{(2 n) !} t^{2 n}=-\frac{1}{16} \sum_{n=1}^{\infty} \frac{b_{n}(2 t)^{2 n}}{(2 n) !},
\end{aligned}
$$

where

$$
b_{n}=(n-4) 4^{2 n}+\left(6 n^{2}+11 n-4\right) 2^{2 n}-4\left(18 n^{2}-15 n-2\right) .
$$

Since $b_{1}=b_{2}=0, b_{3}=756$ and for $n \geq 4$,

$$
\begin{aligned}
b_{n} & \geq\left(6 n^{2}+11 n-4\right) 2^{8}-4\left(18 n^{2}-15 n-2\right) \\
& =4\left(366 n^{2}+719 n-254\right)>0
\end{aligned}
$$

we have $h_{1}(t)<0$ for $t>0$, so is $h^{\prime}(t)$. This leads to $h(t)<\lim _{t \rightarrow 0} h(t)=0$, which proves the second inequality of (20) holds for $t>0$.

Remark 6. Due to

$$
\begin{aligned}
L(a, b) & =\frac{a-b}{\ln a-\ln b}=\int_{0}^{1} a^{s} b^{1-s} d s \\
T Q(a, b) & =\frac{2}{\pi} \int_{0}^{\pi / 2} a^{\cos ^{2} \theta} b^{\sin ^{2} \theta} d \theta=\frac{1}{\pi} \int_{0}^{1} a^{s} b^{1-s}(s(1-s))^{-1 / 2} d s,
\end{aligned}
$$

the referee introduces a new family of means $L_{\alpha}(a, b)$ defined for $\alpha>0$ by

$$
L_{\alpha}(a, b)=\frac{\Gamma(2 \alpha)}{\Gamma(\alpha)^{2}} \int_{0}^{1} a^{s} b^{1-s}(s(1-s))^{\alpha-1} d s .
$$

The referee also gives an interesting relation between this new mean and the modified Bessel functions of the first kind:

$$
\frac{L_{\alpha}(a, b)}{\sqrt{a b}}=\Gamma\left(\alpha+\frac{1}{2}\right)\left(\frac{t}{2}\right)^{1 / 2-\alpha} I_{\alpha-1 / 2}(t), \quad t=\ln \sqrt{\frac{a}{b}} .
$$

It is easy to check that

$$
\lim _{\alpha \rightarrow 0} L_{\alpha}(a, b)=\frac{a+b}{2}
$$


and

$$
\begin{aligned}
L_{\alpha}(a, b) & =\frac{\Gamma(2 \alpha)}{\Gamma(\alpha)^{2}} \int_{0}^{1} a^{s} b^{1-s}(s(1-s))^{\alpha-1} d s \\
& <\frac{\Gamma(2 \alpha)}{\Gamma(\alpha)^{2}} \int_{0}^{1}(s a+(1-s) b)(s(1-s))^{\alpha-1} d s=\frac{a+b}{2} .
\end{aligned}
$$

However, more problems remain to be researched on this new family of means, for example: (i) checking the monotonicity of this mean with respect to the parameter $\alpha$; (ii) finding the lower and upper bounds for this mean in terms of elementary means; (iii) comparing this new mean with others.

Author Contributions: Formal analysis, Z.-H.Y. and J.-F.T.; Funding acquisition, Y.-R.Z.; Methodology, Z.-H.Y.; Supervision, Z.-H.Y.; Writing—original draft, Z.-H.Y.; Writing—review \& editing, J.-F.T. and Y.-R.Z. All authors have read and agreed to the published version of the manuscript.

Funding: This research was funded by the Fundamental Research Funds for the Central Universities under Grant MS117 and Grant 2015ZD29.

Acknowledgments: The authors would like to express their sincere thanks to the editors and reviewers for their great efforts to improve this paper.

Conflicts of Interest: The authors declare no conflict of interest.

\section{References}

1. Watson, G.N. A Treatise on the Theory of Bessel Functions; Cambridge University Press: Cambridge, UK, 1922.

2. Yang, Z.-H.; Tian, J.-F. A class of completely mixed monotonic functions involving the gamma function with applications. Proc. Am. Math. Soc. 2018, 146, 4707-4721. [CrossRef]

3. Yang, Z.-H.; Tian, J.-F.; Ha, M.-H. A new asymptotic expansion of a ratio of two gamma functions and complete monotonicity for its remainder. Proc. Am. Math. Soc. 2020, 148, 2163-2178. [CrossRef]

4. Yang, Z.-H.; Tian, J.-F.; Wang, M.-K. A positive answer to Bhatia-Li conjecture on the monotonicity for a new mean in its parameter. Rev. R. Acad. Cienc. Exactas Fís. Nat. Ser. A Mat. RACSAM 2020, 114, 126. [CrossRef]

5. Neuman, E. Inequalities involving modified Bessel functions of the first kind. J. Math. Anal. Appl. 1992, 171, 532-536. [CrossRef]

6. Baricz, Á. Turán type inequalities for modified Bessel functions. Bull. Aust. Math. Soc. 2010, 82, $254-264$. [CrossRef]

7. Segura, J. Bounds for ratios of modified Bessel functions and associated Turán-type inequalities. J. Math. Anal. Appl. 2011, 374, 516-528. [CrossRef]

8. Kokologiannaki, C.G. Bounds for functions involving ratios of modified Bessel functions. J. Math. Anal. Appl. 2012, 385, 737-742. [CrossRef]

9. Hornik, K.; Grün, B. Amos-type bounds for modified Bessel function ratios. J. Math. Anal. Appl. 2013, 408, 91-101. [CrossRef] [PubMed]

10. Baricz, Á. Bounds for Turánians of modified Bessel functions. Expo. Math. 2015, 2015, 223-251. [CrossRef]

11. Yang, Z.-H.; Zheng, S.-Z. Sharp Bounds for the Ratio of Modified Bessel Functions. Mediterr. J. Math. 2017, 14, 169. [CrossRef]

12. Toader, G. Some mean values related to the arithmetic-geometric mean. J. Math. Anal. Appl. 1998, 218, 358-368. [CrossRef]

13. Qi, F.; Shi, X.-T.; Liu, F.-F.; Yang, Z.-H. A Double Inequality for an Integral Mean in Terms of the Exponential And Logarithmic Means. ResearchGate Research. Available online: http:/ /dx.doi.org/10.13140/RG.2.1.2353. 6800 (accessed on 1 June 2015).

14. Qi, F.; Shi, X.-T.; Liu, F.-F.; Yang, Z.-H. A double inequality for an integral mean in terms of the exponential and logarithmic means. Period Math. Hung. 2017, 75, 180-189. [CrossRef]

15. Lin, T.-P. The power mean and the logarithmic mean. Am. Math. Mon. 1974, 81, 879-883. [CrossRef]

16. Stolarsky, K.B. Generalizations of the Logarithmic Mean. Math. Mag. 1975, 48, 87-92. [CrossRef]

17. Páles, Z. Inequalities for differences of powers. J. Math. Anal. Appl. 1988, 131, 271-281. [CrossRef] 
18. Tian, J.; Zhu, Y.-R.; Cheung, W.-S. N-tuple Diamond-Alpha integral and inequalities on time scales. N-tuple Diamond-Alpha integral and inequalities on time scales. Rev. R. Acad. Cienc. Exactas Fís. Nat. Ser. A Mat. RACSAM 2019, 113, 2189-2200. [CrossRef]

19. Hu, X.-M.; Tian, J.-F.; Chu, Y.-M.; Lu, Y.-X. On Cauchy-Schwarz inequality for N-tuple Diamond-Alpha integral. J. Inequal. Appl. 2020, 2020, 8. [CrossRef]

20. Yang, Z.-H. On the log-convexity of two-parameter homogeneous functions. Math. Inequal. Appl. 2007, 10, 499-516. [CrossRef]

21. Yang, Z.-H. On the monotonicity and log-convexity of a four-parameter homogeneous mean. J. Inequal. Appl. 2008, 2008, 49286. [CrossRef]

22. Alzer, H. Ungleichungen für Mittelwerte. Arch. Math. 1986, 47, 422-426. [CrossRef]

23. Yang, Z.-H.; Chu, Y.-M. On approximating the modified Bessel function of the first kind and Toader-Qi mean. J. Inequal. Appl. 2016, 2016, 40. [CrossRef]

24. Yang, Z.-H.; Chu, Y.-M.; Song, Y.-Q. Sharp bounds for Toader-Qi mean in terms of logarithmic and identric means. Math. Inequal. Appl. 2016, 19, 721-730. [CrossRef]

25. Yang, Z.-H.; Chu, Y.-M. A sharp lower bound for Toader-Qi mean with applications. J. Funct. Space. 2016, 2016, 4165601. [CrossRef]

26. Qian, W.-M.; Zhang, X.-H.; Chu, Y.-M. Sharp bounds for the Toader-Qi mean in terms of harmonic and geometric means. J. Math. Inequal. 2017, 11, 121-127. [CrossRef]

27. Biernacki, M.; Krzyz, J. On the monotonicity of certain functionals in the theory of analytic functions. Ann. Univ. Mariae Curie-Sklodowska 1955, 9, 135-147.

28. Yang, Z.-H.; Chu, Y.-M.; Wang, M.-K. Monotonicity criterion for the quotient of power series with applications. J. Math. Anal. Appl. 2015, 428, 587-604. [CrossRef]

29. Wang, M.-K.; Chu, Y.-M.; Song, Y.-Q. Asymptotical formulas for Gaussian and generalized hypergeometric functions. Appl. Math.Comput. 2016, 276, 44-60. [CrossRef]

30. Yang, Z.-H.; Chu, Y.-M. A monotonicity property involving the generalized elliptic integrals of the first kind. Math. Inequal. Appl. 2017, 20, 729-735.

31. Yang, Z.-H.; Zheng, S.-Z. Monotonicity and convexity of the ratios of the first kind modified Bessel functions and applications. Math. Inequal. Appl. 2018, 21, 107-125.

32. Yang, Z.-H.; Tian, J. Sharp inequalities for the generalized elliptic integrals of the first kind. Ramanujan J. 2019, 48, 91-116. [CrossRef]

33. Anderson, G.D.; Vamanamurthy, M.; Vuorinen, M. Monotonicity rules in calculus. Am. Math. Mon. 2006, 113, 805-816. [CrossRef]

34. Pólya, G.; Szegö, G. Problems and Theorems in Analysis I: Series. Integral Calculus, Theory of Functions; Springer: Berlin/Heidelberg, Germany; New York, NY, USA, 1998.

35. Li, Y.-M.; Long, B.-Y.; Chu, Y.-M. Sharp bounds by the power mean for the generalized Heronian mean. J. Inequal. Appl. 2012, 2012, 129. [CrossRef]

36. Abramowitz, M.; Stegun, I.A. Handbook of Mathematical Functions with Formulas, Graphs, and Mathematical Tables; Dover Publications: New York, NY, USA; Washington, DC, USA, 1972.

(c) 2020 by the authors. Licensee MDPI, Basel, Switzerland. This article is an open access article distributed under the terms and conditions of the Creative Commons Attribution (CC BY) license (http://creativecommons.org/licenses/by/4.0/). 